\title{
Immunity to proactive interference is not a property of the focus of attention in working memory
}

\author{
Alicia Ralph • Jade N. Walters • Alison Stevens • \\ Kirra J. Fitzgerald • Gerald Tehan • \\ Aimee M. Surprenant • Ian Neath • Josée Turcotte
}

Published online: 10 November 2010

(C) Psychonomic Society, Inc. 2010

\begin{abstract}
The Focus of Attention (FOA) is the latest incarnation of a limited capacity store in which a small number of items, in this case four, are deemed to be readily accessible and do not need to be retrieved. Thus a corollary of these ideas is that those items in the FOA are always immune to proactive interference. While there is empirical support for instances of immunity to PI in short-term retention tasks that involve memory for four-item lists, there are also many instances in which PI is observed with four-item lists as well as instances where PI and immunity to PI can be shown in the same experiment. In contrast to the FOA assumptions, an alternative cue-based account predicts both the presence of PI and immunity to PI as a function of the relation between the cues available and the particular test. Three experiments contrasted the FOA assumptions and the cue-based approach in a short-term cued recall task in which PI is manipulated by testing whether the presentation of previous, similar items would interfere with immediate recall of three list items. The results indicated that even with very short lists, both PI and immunity to PI could be observed. The PI effects observed
\end{abstract}

\footnotetext{
A. Ralph · J. N. Walters · A. Stevens · K. J. Fitzgerald · G. Tehan University of Southern Queensland, Springfield, QLD, Australia

A. M. Surprenant $\cdot$ I. Neath

Memorial University of Newfoundland,

St. John's, NL, Canada

J. Turcotte

Laurentian University,

Sudbury, ON, Canada

G. Tehan $(\bowtie)$

Department of Psychology, University of Southern Queensland, PO Box 4196, Springfield Central 4300, Australia

e-mail: tehan@usq.edu.au
}

in our experiment are at odds with the FOA approach and are more readily explained using the cueing account.

Keywords Proactive interference $\cdot$ Short-term memory Focus of attention. Serial recall $\cdot$ Cued recall

\section{Introduction}

There is widespread agreement in the memory literature that there is a limit on the amount of information that humans can retain and utilise at any one moment. The specific nature of this limitation has received considerable attention with one general solution to the problem involving the invocation of a special limited capacity storage mechanism of one type or another. This limited capacity store has been given various labels over the years, for example, Short-term Store (Atkinson \& Shiffrin, 1968), Primary Memory (Waugh \& Norman, 1965), the Phonological Loop (Baddeley, 1986), and the Focus of Attention (Cowan, 1999, 2001). All these approaches make an implicit (and sometimes explicit) assumption that the items in the special store are readily available for processing and do not have to be retrieved. A corollary of such an assumption, again explicitly made in some models (Cowan, 2001; Wickens, Moody, \& Dow, 1981), is that those items in the special store are immune to interference from items outside this special store. That is, these items are immune to the effects of proactive interference (PI). Thus, one clear implication of these assumptions is that there should be a link between the capacity of the special store and the observation of PI. If the number of to-beremembered items is within the capacity of the special store, then performance should be immune to PI. However, if capacity is exceeded or the contents of the special store are not accessed, PI should be readily apparent. 
While the above instances all assume a limited capacity store, not all agree upon the actual capacity of the store. Thus, initial estimates of the capacity of the short-term store were seven plus or minus two chunks (Miller, 1956); the capacity of PM was often determined to be in the region of four to five items (Tulving \& Patterson, 1968; Waugh \& Norman, 1965; Watkins, 1974); the capacity of the phonological loop was equivalent to what could be rehearsed in approximately two seconds (Baddeley, Thomson, \& Buchanan, 1975), which translated to between four and seven items depending upon the material being remembered; and the capacity of the Focus of Attention is deemed to be no more than four items (Cowan, 2001). If these short-term stores protect against PI, PI should be apparent with list lengths greater than seven items, it may be observable with list lengths between four and seven items, but should not be observable with list lengths of four or below.

\section{The embedded processes model}

Before examining the data on short-term PI effects, we want to explore one of the above models that explicitly makes the link between capacity and immunity to PI and assumes capacity estimates at the lower end of the spectrum. Cowan's $(1999,2001)$ embedded processes model represents the latest version of the special store. Cowan first argued that since processes such as coding require contact with representations in the LTS, information from sensory storage must immediately make contact with the LTS. Cowan $(1995,1999)$ thus proposed a unitary-store model with three embedded components. In this model, LTM underlies all memory and is assumed to be unlimited in capacity. The representations in LTM that have an increased strength of activation above baseline levels are deemed to be in activated memory (the second component) which can be equated with STM or working memory. These activated representations are subject to rapid decay unless they are brought into the focus of attention (FOA), the third component, a subset of activated memory in which representations are maximally activated and immediately available for processing. An item in either activated memory or LTM will enter the FOA provided that it is novel or that the subject chooses to attend to it. As well as this memory store, Cowan (1999) proposed that there is an automatic attention orienting system and a central executive, which functions in a similar manner to the central executive proposed by Baddeley and Hitch (1974). Thus, it is the central executive that directs the process of voluntary attention, during which items are intentionally placed in the FOA.

Cowan (2001) reviewed an extensive number of experiments on capacity limitations and concluded that the classic seven plus or minus two (Miller, 1956) was an overestimate because it was based on studies that failed to prevent participants from rehearsing or chunking items together. Due to observations such as sharp declines in performance after about four items in short-term retrieval tasks, and errorless performance on tasks with less than this, Cowan (2001) concluded that the capacity of the FOA was actually four items, plus or minus one. Items are lost from the FOA by the entry of additional information into the store after capacity has been reached. Thus, if attention is shifted from one set of items to another, the first set will be lost from the FOA, but will remain in activated memory for some period during which the item return to baseline levels of activation (LTM), unless they are brought back into the FOA.

According to the model, retrieval involves placing items from either LTM or activated memory into the FOA. Whilst this retrieval process is prone to the effects of interference, once in the FOA items are assumed to have already been retrieved, and so are immediately available for processing. Being in the FOA effectively isolates those items from potential competitors and thus those items in the FOA are immune to effects of interference.

An important conclusion from the above review is that items within the FOA should be immune to PI, whereas items outside the FOA should be prone to PI. Hence, on a task where participants are required to remember only a few items, performance should reflect immunity to PI. However, on a task where the capacity of the FOA has been exceeded, performance should show the effects of PI.

There is substantial empirical support for the FOA assumptions. Immediate tests of subspan lists are largely immune to PI when correct recall is the dependent measure (Dempster \& Cooney, 1982; Humphreys \& Tehan, 1992; Sanders \& Willemsen, 1978; Tehan \& Humphreys, 1995) or when reaction time is used in recognition paradigms (Cowan, Johnson, \& Saults, 2005; Halford, Maybery, \& Bain, 1988; Wickens et al., 1981). Two studies in particular are relevant to the linkage between capacity and PI. Halford, Maybery, and Bain (1988) manipulated PI within the context of the Sternberg scanning paradigm. The important aspect of this research was that the memory search set consisted of four, six, eight or ten items on each trial. They found that on an immediate test, PI was observed for those trials that involved set sizes of six, eight and ten. However, immunity to PI was found for the trials with a set size of four items. Cowan, Johnson, and Saults (2005) replicated this study using set sizes of three, four, six and eight. They too found PI in lists of six and eight items, and immunity to PI in lists of three and four items. They concluded that this finding is consistent with the hypothesis that adults can keep about four items active in the FOA at one time. 


\section{Evidence of proactive interference}

In spite of the data supporting the FOA approach, PI has been found in immediate recall of short lists of three or four items in recognition tasks (Branelley, Tehan, \& Humphreys, 1989; Carroll, Jalbert, Penney, Neath, Surprenant, \& Tehan, 2010; Hanley \& Scheirer, 1975; Jonides, Marshuetz, Smith, Reuter-Lorenz, \& Koeppe, 2000; Monsell, 1978) and in recall tasks (Tehan \& Humphreys, 1995, 1998; Tolan \& Tehan, 1999). We focus on the Tehan and Humphreys paradigm, rather than the probe recognition studies, because in a number of experiments where recall of four items was tested, both immunity to PI and PI have been demonstrated within the same experiment.

Tehan and Humphreys developed a short-term cued recall task, specifically designed to test for PI effects. Each trial consisted of either one block or two blocks of four words. Memory for the most recent block was tested via a category cue. On all trials one of the four items in the most recent block was an instance of the category and the participants' task was to recall the target item. The critical trials in the experiment were the two-block trials. The twoblock trials consisted of four items in each block and the blocks were separated by an exclamation mark. The exclamation mark served as was an indicator to the participant that they were to forget the items that they had just seen and concentrate on the next block of items because it was the memory for these items that would be tested. All items in a trial were unrelated to each other in terms of category membership except on the interference trials where one of the items in the first to-be-forgotten block came from the same category as the target item in the second block. Thus a typical interference trial would appear as: meal dog engine road! image cat gate starch ANIMAL. The correct response in this trial would be cat. A no interference trial would be identical save that $d o g$ in block1 would be replaced by a filler item like cloud. In this task PI could be reflected in either (or both) changes in the level of recall of the target item or in recall of the block- 1 foil instead of the target.

Using this task Tehan and Humphreys (Experiment 3; 1995) found that PI occurred after a two-second delay, but not on an immediate test. That is, on the immediate test, the target item was recalled equally well in the interference and no interference conditions and the block-1 foil was very infrequently recalled. However, on the delayed test, target recall was reduced in the interference trials and recall of the block-1 foil became a relatively frequent source of error.

In two subsequent experiments that explored the generality of immunity to PI in the cued recall task, participants were tested for immediate recall of list items in which the target and the foil were rhymes of each other. Thus in one experiment the target might have been cat, the foil hat and the cue was an ending cue ( AT). In a second experiment the target and foil were Rhyming words instances of taxonomic categories (e.g. cat and rat) and were tested using a category cue (ANIMAL). In both experiments, on immediate testing, target recall was poorer in the interference trials than the control trials, and there were a relatively large number of instances in which the foil was recalled instead of the target, regardless of whether the probe was a category cue or an ending cue. Thus, PI was observed on an immediate test of a four-item list when the target and foil rhymed.

The authors suggested that immunity to PI may be related to discriminative information that is provided by transient phonemic codes. Thus in the cued recall task described earlier, the category cue is assumed to elicit the semantic representations of both the target and foil on the interference trials; that is, there is the opportunity for PI to occur. It is phonemic coding however, which adds the critical information to enable the discrimination of the target from the foil. If the phonemic code is available to support the target, but not the foil, then the presence of discriminative phonemic information allows the participant to select the target and reject the foil.

In a final test of these ideas, Tehan and Humphreys (1995); Experiment 5; see also Tolan \& Tehan, 1999) varied the modality of the first and second block to manipulate presence or absence of phonological codes for the block-1 foil. Thus, the first block was read aloud and the second block read silently, or vice versa. The logic here was that phonological codes are transient and will be stronger for the second block than the first. In addition, reading items aloud boosts phonological codes. In the case of a SILENT-ALOUD configuration, the phonological codes for the target will be very strong and immunity to PI should result. In the ALOUD-SILENT configuration it was argued that when the first block is read aloud, the phonological codes for these items are boosted and still available to some degree at recall. As such, the strength of phonological codes from the first and the second block are about equal at the point of recall, in that the boost from reading aloud the first block is roughly cancelled by the decline over time. Thus even though target and foil might not rhyme, there might well be roughly equivalent phonological information available for both target and foil, leading to the target and the foil competing for recall. The data were consistent with these assumptions in that there was no PI in the SILENT-ALOUD condition but strong PI effects in the ALOUD-SILENT condition.

Tehan and Humphreys (1995) suggested that there are three conditions in which PI should be observed: (1) when there is no phonemic information about the target item as is the case with delayed tests; (2) when there is phonemic information but it fails to discriminate between the target 
and interfering items as is the case when target and foil rhyme; and (3) when there is phonemic information for both target and interfering items, as when the first block is read aloud and the second silently (Tehan \& Humphreys, 1995; Tolan \& Tehan, 1999). The only time that immunity to PI should be observed is when there is phonemic information for only the target item and this information discriminates between the target and any interfering items.

In order to provide an additional test of the third prediction that PI would be observed if there was phonemic information for the foil, Tehan and Humphreys (1998) explored whether or not the phonemic features of the foil could be provided by another list item that was a rhyme of the foil. The cued recall task was again used, but with three conditions: the standard control and interference conditions, and an interference condition in which a rhyme of the foil was included in the second block of words. Thus a typical interference-plus-rhyme trial would appear as: meal dog engine road! image cat log starch $A N I M A L$. The results indicated that correct target recall was significantly lower in the interference-plus-rhyme condition than the control condition. However, there was no difference between the standard-interference and the control conditions. In addition, there were significantly more instances in which the foil was incorrectly recalled in the interference-plus-rhyme condition than in the standard-interference condition. Thus, as predicted, immunity to PI was found in the absence of a rhyme, and PI was found when a rhyme of the foil was included. The generality of this finding has been subsequently well established using alternative means of providing phonemic support for the foil (Tolan \& Tehan, 2002).

The cued recall experiments show that both immunity to PI and the presence of PI can be readily observed on an immediate test where the memory load is four words. Immunity to PI with a four-item memory load is readily accounted for by Cowan's embedded processes model, but the presence of PI is less readily explained. Tehan and Humphreys' cue plus codes account predicts when PI will and will not be observed so at face value the latter account is more parsimonious. However, because the capacity of the FOA is four plus or minus one items, it is possible that PI was observed in the Tehan and Humphreys (1998) experiment because a four-item list exceeded the capacity of the FOA for some people or on some trials. The FOA approach would be less tenable if PI could be found with a memory load that was less than four items.

\section{Experiment overview}

In the experiments that follow, PI is manipulated using Tehan and Humphrey's two-block directed forgetting procedure in which the two blocks are separated by a one-second interval in which the directed forgetting instruction is given. Empirical evidence suggests that items are lost from the FOA within this timeframe (Oberauer, 2002, 2005).

In the first experiment the cued recall version of the task is used with a memory load of three words. In the second and third experiments the same basic task is again used but serial recall rather than cued recall is used to examine the generality of PI effects. Immediate serial recall has been the paradigm of choice for much of the recent theoretical developments in short-term/working memory but given the prominence of the task, it is somewhat surprising that PI has not been systematically studied in this task. Thus, in Experiments 2 and 3, the two-block design is used but serial recall of the second block is required. Phonological information is manipulated either by having all the items in the block being phonologically dissimilar to each other (discriminative information) or all items in the block rhyme with each other (non-discriminative information). In Experiment 2 there are five items in the second block that have to be recalled in order and in Experiment 3 there are three items for recall. Varying list length leads to different predictions of the two approaches. The phonemic codes approach assumes that list length is irrelevant and that the presence or absence of PI will depend upon the availability of discriminative phonological information, such that in each experiment it will be possible to observe both PI and immunity to PI in the same experiment. In contrast the embedded processes model predicts that immunity to PI should be observed with three-item lists (Experiments 1 \& 3), but PI should be observed with five-item lists (Experiment 2).

\section{Experiment 1}

In this experiment the cued task was modified so that on the trials of interest participants would be required to remember only three items instead of four, which from the FOA perspective should be well within the capacity limit of normal, healthy adults. A straightforward prediction of the embedded processes model is immunity to PI for all conditions. On the other hand, the phonemic codes account makes the predictions that immunity to PI will occur in the standard-interference condition but that PI will be observed in an interferenceplus-rhyme condition. That is, there will be a decrement in correct target recall compared to the no-interference condition and that there would be an increase in block-1 intrusion errors relative to the standard interference condition. 


\section{Method}

Participants

Thirty participants (11 male) volunteered or were given partial course credit to participate in the experiment. All participants reported having normal or corrected to normal vision and had English as their first language.

\section{Materials}

The experiment consisted of 40 one- or two-block trials. The 10 one-block trials were included to ensure that participants attended to the first block in each trial. These blocks always contained four items (one target and three randomly assigned filler items). In these trials, the target item appeared in position- 1 on half the trials, and position-4 on the other half. The materials used in these trials had no overlap with the materials in the critical two-block trials.

Proactive interference was manipulated in the 30 twoblock trials. In these trials, the first block always contained four items and the second block contained three. There were three experimental conditions in the experiment each with ten trials. In the no-interference trials the first block consisted of only filler items, and a target appeared in the second block. In the standard-interference trials an interfering foil appeared in the first block and the target in the second. In the interference-plus-rhyme trials an interfering foil appeared in the first block, and a rhyme of the foil and the target appeared in the second block.

Thirty targets and interfering foils were selected from those used in Tehan and Humphreys' (1998) experiment. The filler items were selected from categories in Van Overschelde, Rawson and Dunlosky's (2004) category norms, such that there was no overlap in category membership between filler and critical items. Each filler item was a one or two syllable, concrete noun and as such had the same characteristics as the targets and foils. The rhyme of each foil was selected from Walling, McEvoy, Oth, and Nelson's (1983) rhyme category norms. These items were also chosen to have the same basic characteristics as the other items and had no category membership overlap with the critical items.

In creating the trials for each participant, foils and their corresponding targets and rhymes were randomly assigned to the three experimental conditions. In both types of interference trials the target and foil were always separated by three items (two fillers and the block separator) such that on half the trials the foil appeared in position- 2 and the target in position-1, of their respective blocks and on the other half, the foil appeared in position- 3 and the target in position-2, of their respective blocks. In the no-interference trials only the target item appeared among six fillers and it also appeared in position- 1 on half the trials and position- 2 on the other half. In the interference-plus-rhyme condition the rhyme also appeared in either the first or second serial position in the second block, such that it was always adjacent to the target. This meant that the critical items were never in the first position in the first block, and never in the last position in either block.

The order of the 40 trials was randomised, and the above procedure for creating the trials was conducted for each participant, such that 30 unique sets of trials were created.

\section{Procedure}

Upon consenting to participate in the experiment, participants were told that they would be studying lists of words for cued recall. These words would be presented in either one or two blocks of either three or four items. Moreover, at any time they would only have to remember the most recent block of items that they had seen. Thus, if the trial was a two-block trial, once they knew that the second block was going to appear, they could forget the first block and concentrate on remembering the second block. However, because the order of trials would be randomised, they would not be able to predict whether the trial would be a one- or two-block trial. It would therefore be best to treat each trial as if it were a one-block trial until they learned otherwise. Participants were instructed that at the beginning of each block they would see either the word ALOUD or SILENT in capital letters which was a cue to indicate whether they needed to read the memory items aloud or silently. In fact each trial began with the word ALOUD, and in the two-block trials, blocks were separated by the word SILENT. Thus, participants were always required to read the first block in a trial aloud and the second block silently.

Trials began with the word ALOUD presented on the screen for two seconds. The block-1 items were then presented individually in lower case at a rate of one word per second. In the two-block trials the word SILENT then appeared for one second, followed by the three block-2 items at a rate of one word per second. Immediately after each trial ended, a category cue appeared in upper case on the computer monitor for two seconds. Participants were instructed to then verbally recall the item from the most recent block that was an instance of this category. Participants then began a new trial by pressing the space bar on the keyboard when they were ready to continue.

The experimenter recorded the participant's verbal responses on a hardcopy of their input file. There were four types of responses that a participant could make: correct target recall, foil recall (block-1 intrusion), omission, or extra-list intrusion. 


\section{Results}

On the one-block trials target recall was $98.2 \%$ accurate. Participants clearly attended to the first block.

The main results of the experiment are presented in Fig. 1. With the primary measure of target recall and block1 intrusions, it is clear that the interference plus rhyme condition led to fewer target recalls and more block-1 intrusions compared to the other two conditions which do not differ when it comes to target recall.

The analysis of target recall in the block- 2 trials showed that there were significant differences in correct target recall between the three experimental conditions, $F(2,58)=5.19$, $\eta^{2}=0.15, M S E=1.04, p<0.05$. Planned contrasts showed that target recall was significantly lower in the interference-plus-rhyme condition than in the nointerference condition, $F(1,29)=7.82, \eta^{2}=0.21, M S E=$ 2.25, $p<0.01$. There were no significant differences in target recall between the no-interference condition and the standard-interference condition, $F(1,29)=0.11, M S E=$ $1.24, p>0.05$.

Because so few Block-1 errors were made in some of the conditions a parametric statistical analysis of the data is inappropriate. Consequently a Wilcoxon non-parametric test showed that there were significantly more intrusions made in the interference-plus-rhyme condition than the standard-interference condition, $Z=3.10, p<0.002$. Table 1 shows the frequency with which block-1 errors were made. In the standard interference condition $73 \%$ of participants did not make a block-1 intrusion, whereas in the interference plus rhyme condition, only $43 \%$ made no intrusions. Moreover, making multiple intrusions was much more likely in the interference plus rhyme condition than the standard interference condition. Thus, block-1 intrusions in the interference plus rhyme condition were wide-spread among participants and relatively frequent.

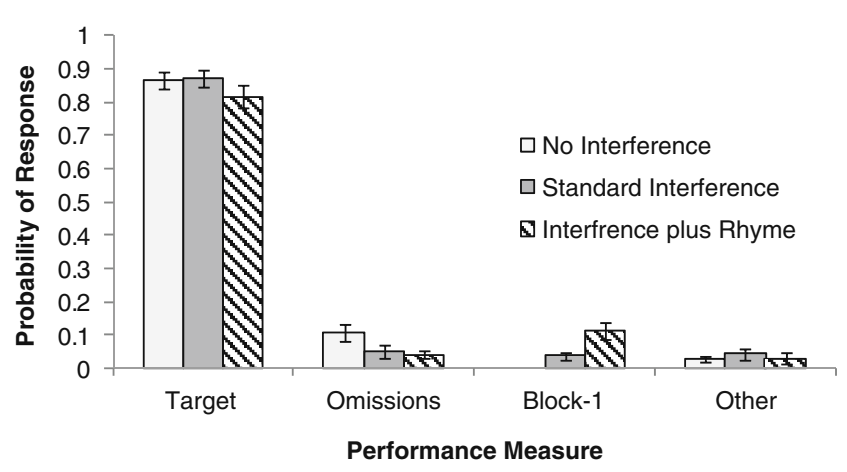

Fig. 1 Mean number of correct target recall, omission, extra-list intrusion, and block-1 intrusion responses made in each interference condition. Error bars show the SEM

\section{Discussion}

The results are quite straightforward: On the two-block trials, immunity to PI is observed in the standard interference condition but in the interference plus rhyme condition PI is observed in that there is both a decrement in target recall and an increase in the number of block-1 intrusions. All that differs between the two interference conditions is that one of the filler items changes from being unrelated to the foil to a rhyme of the foil.

These latter findings are consistent with the cues plus code approach but are problematic for the embedded processes model because with a memory load of only three items, the capacity of the FOA should not be exceeded. Moreover, according to the FOA approach, having discriminative phonological information available at recall should not be important, since items within the FOA have already been activated and so do not need to be discriminated from other items.

There are some methodological factors that may have influenced overall levels of performance (differential category dominance of the target and foil, serial position of targets and fillers) but none of these factors are able to account for the differential effects of the presence of a rhyme. Moreover, a number of methodological factors should make discrimination problems easier. For example, the first block always contained four items and was read aloud, the second block contained three items and was read silently. Thus list length and modality provided two good cues that would allow participants to discriminate the target from the foil and thereby minimise the effects of PI. The fact that PI emerged under conditions where the task was relatively easy attests to the role of encoding and retrieval interactions over the short-term. Short-term recall is not a simple matter of reading out items from a limited capacity store.

\section{Experiment 2}

The cued recall task requires that a person remember that an item was on a list but not the item's position. In this experiment, we changed the memory test to one which explicitly required item and order information. In this experiment the two-block interference design was again used, but with a different number of items: the first to-beforgotten block consisted of four items and the second tobe-remembered block consisted of five items which have to be recalled in order.

We acknowledge that proponents of the FOA approach might argue that five items exceeds the capacity of the focus of attention and thus the predictions we ascribe to the FOA may not be all that strong. However, we began with 
Table 1 Frequency of block-1 intrusions in three experiments

\begin{tabular}{|c|c|c|c|c|c|c|c|c|}
\hline & & \multicolumn{7}{|c|}{ Number of intrusions } \\
\hline & & 0 & 1 & 2 & 3 & 4 & 5 & $6+$ \\
\hline \multicolumn{9}{|l|}{ Experiment 1} \\
\hline Standard interference & & 22 & 6 & 1 & 1 & & & \\
\hline Interference plus rhyme & & 13 & 6 & 6 & 2 & 1 & & \\
\hline \multicolumn{9}{|l|}{ Experiment 2} \\
\hline Rhyming words-Nonrhyming words & & 13 & 5 & & & & & \\
\hline Nonrhyming words-Nonrhyming words & & 13 & 5 & & & & & \\
\hline Nonrhyming words-Rhyming words & & 18 & 1 & & & & & \\
\hline Rhyming words-Rhyming words & & 0 & 3 & 3 & 1 & 2 & 2 & 7 \\
\hline \multicolumn{9}{|l|}{ Experiment 3} \\
\hline \multirow[t]{3}{*}{ Rhyming words-Nonrhyming words } & Part A & 19 & & 1 & & & & \\
\hline & Part B & 18 & 2 & & & & & \\
\hline & Part C & 20 & & & & & & \\
\hline \multirow[t]{3}{*}{ Nonrhyming words-Nonrhyming words } & Part A & 17 & 3 & & & & & \\
\hline & Part B & 18 & 2 & & & & & \\
\hline & Part C & 20 & & & & & & \\
\hline \multirow[t]{3}{*}{ Nonrhyming words-Rhyming words } & Part A & 19 & 1 & & & & & \\
\hline & Part B & 19 & 1 & & & & & \\
\hline & Part C & 17 & 2 & 1 & & & & \\
\hline \multirow[t]{3}{*}{ Rhyming words-Rhyming words } & Part A & 3 & 6 & 8 & 2 & 1 & & \\
\hline & Part B & 9 & 6 & 2 & 2 & 1 & & \\
\hline & Part C & 6 & 7 & 5 & 1 & 1 & & \\
\hline
\end{tabular}

five-item lists in order to determine the accuracy of the predictions of the cue-based account. Given a successful outcome, we then conducted Experiment 3 in which we reduced the number of items to below the capacity of the FOA. Note that the cue-based account makes the same predictions for both experiments.

There were eight conditions in the experiment in which the materials were either a block of letters, a block of nonrhyming words or a block of rhyming words. The eight combinations tested were as follows where the first label in each pair represents the materials used in the first block and the second label indicates the materials used in the second block: Nonrhyming words-Letters, LettersLetters, Letters-Nonrhyming words, Nonrhyming wordsNonrhyming words, Rhyming words-Nonrhyming words, Letters-Rhyming words, Nonrhyming words-Rhyming words, and Rhyming words-Rhyming words. From the cueing perspective, the three different types of material could be used as retrieval cues such that PI has the potential to influence performance only when the materials in the first and second block were the same. Thus, there were three possible conditions where PI might emerge, the LettersLetters, Nonrhyming words-Nonrhyming words, and Rhyming words-Rhyming words conditions.

On the basis of phonological codes the expectation was that any time rhyming words were being recalled the standard decrement in recall would be observed. That is, when the second block contained rhyming words, intra-list discrimination would be difficult and thus recall would be substantially worse than when the items were nonrhyming. However, the cue plus codes approach predicts that in addition to intra-list discrimination problems, recall in the Rhyming words-Rhyming words condition should also suffer from inter-list discrimination difficulties. Thus given that there was the potential for PI and the phonological information did not uniquely support the second block items this should result in an additional decrement in recall. In the other conditions where there is potential for PI, the available phonological information will allow for the block-2 items to be easily discriminated from the block-1 competitors.

Thus the specific predictions are that the FOA approach predicts that all conditions should show PI. The phonemic codes approach makes the prediction that PI will be observed only on the Rhyming-Rhyming trials.

\section{Method}

Participants

Nineteen introductory-level students volunteered or were given partial course credit to participate in the experiment. 
All participants reported having normal or corrected to normal vision and had English as their first language.

\section{Materials}

In this experiment subjects studied a unique set of 100 trials of which 20 were one-block trials and the remaining 80 were two-block trials. For the one-block trials, ten trials consisted of a single block of four letters and ten trials consisted of a single block of four nonrhyming words. These one-block trials were identical for each subject.

Eighty 2-block trials were constructed for each participant. There were ten trials of each of the following eight types: Nonrhyming words-Letters, Letters-Letters, LettersNonrhyming words, Nonrhyming words-Nonrhyming words, Rhyming words-Nonrhyming words, LettersRhyming words, Nonrhyming words-Rhyming words, and Rhyming words-Rhyming words.

To create the materials for the words in the two block trials, nine items from each of 60 different rhyme categories were selected from the South Florida rhyme norms (Walling et al., 1983). The items were one syllable, mostly concrete nouns between three and six letters long.

The first step in creating the two-block trials was to create separate word pools for non-rhyming and rhyming trials and to produce letter sequences. Thirty letter sequences were created by randomly sampling nine consonants from the letter pool which consisted of all English consonants except for the letter "y". On each occasion nine letters were sampled and randomly assigned to the nine serial positions (four in the first block and five in the second block) of the sequence. These thirty letterletter sequences formed the basis of the trials that had letters in the second block and ten of the trials remained intact to form the Letter - Letter trials.

Thirty of the rhyme categories were then randomly selected and the nine items within each category were randomly assigned to the nine serial positions in each sequence. These similar-similar sequences formed the basis of the 30 trials that had phonologically similar items in the second block and again ten of these remained intact to form the Rhyming words - Rhyming words trials. The 270 items from the remaining 30 rhyme categories were then randomised before being assigned to the nine serial positions for 30 sequences. These dissimilar-dissimilar sequences formed the basis for the 30 phonologically dissimilar trials and again ten of these sequences remained unaltered to form the Nonrhyming words-Nonrhyming words trials.

The remaining trials were created by swapping the first blocks of the different sequences. To create the ten Nonrhyming-Letter and ten Letter-Nonrhyming trials, the relevant first blocks from ten each of the Letter-Letter and Nonrhyming-Letter sequences were swapped. To create the ten Letters-Rhyming words trials, the first block of letters from the remaining ten letter-letter sequences was paired with the second block of ten of the Rhyming-Rhyming sequences. To create the Nonrhyming words-Rhyming words and the Rhyming words-Nonrhyming words trials, the first block of the remaining ten Rhyming-Rhyming sequences and the ten remaining Nonrhyming-Nonrhyming sequences were swapped.

Having created 80 such lists, the 20 first block trials were then added and the whole sequence of 100 trials was then randomised. Unique sets of trials were created for each subject.

\section{Procedure}

The directed forgetting instructions were equivalent to those in Experiment 1, save that serial order recall of the most recent block was required. Participants read both blocks silently.

Each trial started with the word READY presented in the middle of the screen. The list items were then presented at a rate of one item per second. In the case of two block trials, an exclamation mark (!) appeared for one second after the final item in the first block to indicate that the trial was a two-block trial. At the end of each trial a row of question marks (???) appeared on the screen as a cue for participants to recall the items in the most recent block.

Participants wrote their responses on a prepared answer sheet. The instructions stressed that participants were to recall from left to right and not to edit their responses in any way.

\section{Results}

Recall of the letters on the one-block trials was on ceiling at $96 \%$ correct and recall of the one-block words was $89 \%$.

Performance on the trials in which letters were recalled in the second block was first examined. When the first block contained words, $92.5 \%$ of letters were recalled in their correct position. When the first block contained other letters, $89.5 \%$ of the letters were recalled in their correct position. This difference was not reliable, $t(18)=1.66, p>$ 0.05 . Given this result and the fact that only three people made a block-1 intrusion, there does not seem to be strong evidence for PI in letter recall.

The results of word recall are summarised in Fig. 2. The standard phonological similarity effect is present in the data in that all the trials with Rhyming words in the second block were not as well recalled as the lists containing Nonrhyming words items. This was due primarily to an 


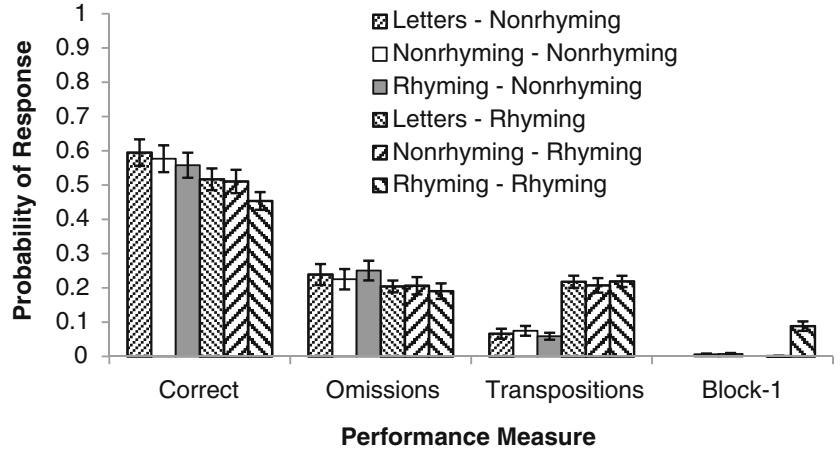

Fig. 2 Mean number of correct recall, omission, transposition, and block-1 intrusion responses made in each interference condition. Error bars show the SEM

increased number of transposition errors. However, there was a further decrement in correct recall performance when the items in the first and second block rhymed with each other. This added decrement was due solely to an increase in block-1 intrusions.

A $2 \times 3$ repeated measures ANOVA with performance collapsed across serial position showed that for block-2 recall when the items were words, nonrhyming words were better recalled than rhyming words, $F(1,18)=24.15, \eta^{2}=$ $0.59, M S E=0.008, p<0.001$. Recall was better under the no-interference condition than the interference condition, $F$ $(2,36)=3.41, \eta^{2}=0.16, M S E=0.008, p<0.044$, and the interaction was not significant. To test our hypotheses two planned one-way repeated measures ANOVAs were conducted; one on the nonrhyming trials and one on the rhyming trials. For the nonrhyming trials there was no difference in recall between the three interference conditions, $F(2,36)=$ $0.87, \eta^{2}=0.05, M S E=0.007, p=0.43$, indicating that the material in the first block had little impact upon target recall. With the rhyming items there was a difference between the three conditions, $F(2,36)=3.15, \eta^{2}=0.15, M S E=0.006$, $p=0.05$, which Fig. 2 indicates is due to a decrement in the Rhyming words-Rhyming words condition.

As far as block-1 intrusions are concerned it is readily apparent in Fig. 2 and Table 1, that in all three replications there were very few block one errors in three of the conditions and that there was a substantial increase in block-1 intrusions when the items in the two blocks all rhymed with each other. A Wilcoxon non-parametric test compared the Rhyming words-Rhyming words lists with the Nonrhyming-Rhyming list. The difference was significant, $Z=3.62, p<0.001$.

According to some models what we refer to as block-1 intrusions could be thought of as additional transposition errors. The most frequent source of order errors is when two adjacent items swap positions. Moreover, items that do not swap adjacent positions tend to migrate to close rather than remote serial positions. If these block-1 intrusions are simply transposition errors, they should fit the same error gradient curve for the within-list order errors.

In the upper panel of Fig. 3 we have plotted the within list order errors for the rhyming and nonrhyming trials and added the block- 1 intrusions for the rhyming lists. These intrusions do not fall on the projection of the within block order errors, instead they cluster around a movement of four serial positions.

\section{Discussion}

The results are very much in line with expectations. We observe the standard phonological similarity effect in that correct recall for block-2 items is worse when the items rhyme than in all the conditions where the block- 2 targets did not rhyme. Moreover, this difference is reflected by a sharp increase in within-block order errors.

We replicate previous experiments when it comes to PI as well. When the block-2 items do not rhyme and are dissimilar to the items in the first block, there is little evidence of PI. Target recall does not appear to be influenced by the material in the first block and block-1 intrusions are very infrequent in the interference conditions. Likewise, there seems to be little evidence for PI with rhyming lists when the first block contains either letters or nonrhyming words. Again there is no difference in target
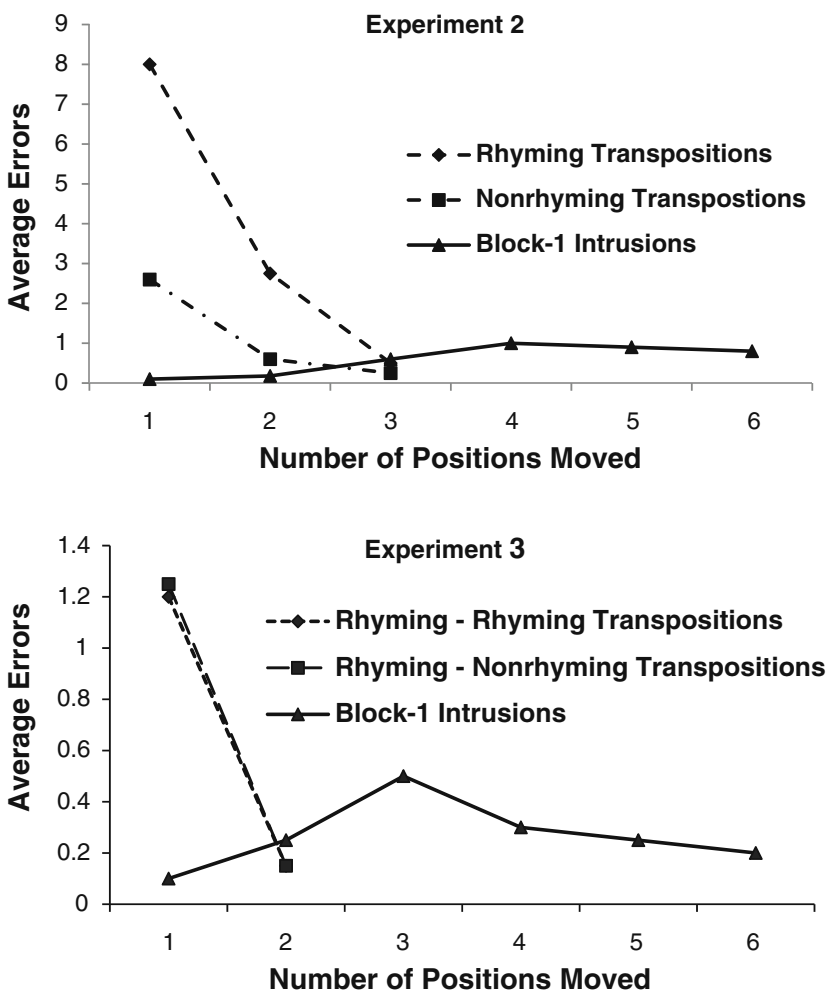

Fig. 3 Error gradients for block-2 transpositions and block-1 intrusions 
recall and block-1 intrusions are again infrequent. Thus, in the instances where phonological information in the second block uniquely specifies the items in the second block, there is very little PI observable. PI effects do become apparent when the items in the first block have similar phonological characteristics to those in the second block. Here there is an added decrement in performance that is attributable primarily to an enhanced number of block-1 intrusions. The block-1 intrusions in the Rhyming wordsRhyming words condition do not appear to be simple order errors. There is a tendency for these words to be recalled in roughly the same position that they occupied in the first block, a pattern that has been found elsewhere (Estes, 1991; Henson, 1998). This pattern also suggests that these block1 intrusions are not simple guesses.

\section{Experiment 3A, B, \& C}

This experiment uses the four different word conditions of Experiment 2, but reduces the list length of the second block from five items to three items. Because the test of the embedded processes model rests on the assumption that the items from the first block are not in the FOA, three conditions were employed to ensure discrimination between the first and second blocks. In Experiment 3A, the standard procedures used in Experiment 2 were used. In Experiment 3B to ensure that participants attended to the instruction to forget the first block and concentrate on the second block, participants were requested to say the word "Beep" when the SILENT instruction appeared as the precursor to the first item in the second block. In Experiment $3 \mathrm{C}$ the pause between blocks was extended from one second to two seconds thus ensuring that at the point of recall a total of five seconds had elapsed since the last item in the first block had been presented (and earlier items rehearsed). Given this timeline, items from the first block should not be in the FOA. In trying to enhance discrimination between the first and second block we risk the possibility of ceiling effects (which the FOA model would predict), but the expectation from the FOA approach would be that PI effects should not have any impact upon performance. The phonemic code account still makes the prediction that PI will be observed when both the first and second blocks contain rhyming words.

\section{Method}

Participants

There were 20 participants in each part of the experiment, giving a total 60 participants.

\section{Materials}

The materials from Experiment 2 were again utilised in this experiment and the lists were constructed in the same way. The two changes involved deleting all conditions in which letters were involved. Thus, the critical conditions involved Nonrhyming words-Nonrhyming words, Rhyming wordsNonrhyming words, Nonrhyming words-Rhyming words, and Rhyming words-Rhyming words combinations. The second change was that the second block of all these trials contained three rather than five items. Thus, in total the participants studied 60 trials, ten trials in each of the above conditions plus 10 one-block trials consisting of four nonrhyming items and 10 one-block trials consisting of four rhyming words. Again the 60 trials were randomised and unique sets of trials were created for each participant.

Procedure

The procedure was identical to that in Experiment 1 in that the first block was read aloud and the second silently and items were presented at the same rate as in Experiment 2. The differences between the different versions of the experiment are that the block separator, SILENT, was presented for one second and was read silently in $3 \mathrm{~A}$ and read aloud in $3 \mathrm{~B}$. In $3 \mathrm{C}$ the separator was read silently but appeared on the screen for two seconds. In all three parts of the experiment participants recalled the list items vocally and the experimenter recorded the output on prepared sheets.

\section{Results and discussion}

\section{One-block trials}

With regards to recall of the one-block trials, the advantage of nonrhyming lists over rhyming lists was significant in each part of the experiment. In part 3A, mean Nonrhyming $=0.89$ and mean Rhyming 0.72, $t(19)=5.06, p<0.001$. In 3B, Nonrhyming $=0.87$ and Rhyming $=0.77, t(19)=3.25$, $p=0.004$; and in $3 \mathrm{C}$, Nonrhyming $=0.92$ and Rhyming $=$ $0.83, t(19)=3.30, p=0.004$.

\section{Correct recall}

The proportion correct in position and the errors made in all three parts of the experiment are presented in the three panels of Fig. 4. Each of the experiment was analysed using a 2 (interference) by 2 (similarity) repeated measures ANOVA. In each replication nonrhyming words were better recalled than rhyming words. In $3 \mathrm{~A}: F(1,19)=14.57, \eta^{2}=$ $0.434, M S E=0.008, p=0.001$; in $3 \mathrm{~B}, F(1,19)=5.21$, 

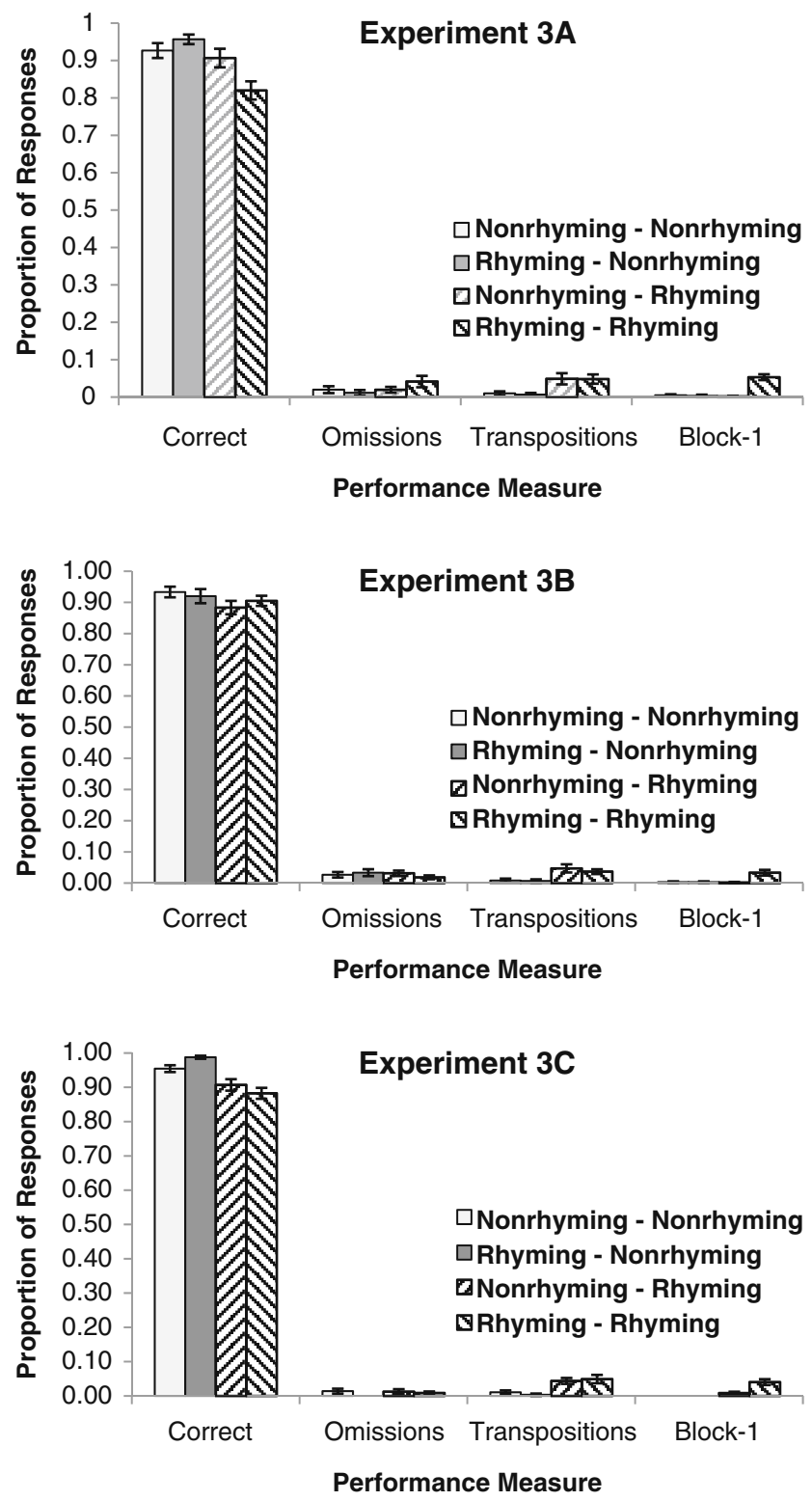

Fig. 4 Mean number of correct recall, omission, transposition, anz block-1 intrusion responses made in each interference condition for Experiment 3A, 3B and 3C. Error bars show the SEM

$\eta^{2}=0.211, M S E=0.004, p=0.034$; and in 3C: $F(1,19)=$ $14.57, \eta^{2}=0.434, M S E=0.008, p=0.001$. The effects of interference were not consistent. In $3 \mathrm{~A}$ there was an overall interference effect, $F(1,19)=15.88, \eta^{2}=0.117, M S E=$ $0.004, p=0.001$, which was replicated in $3 C, F(1,19)=$ 8.00, $\eta^{2}=0.296, M S E=0.003, p=0.011$, but was not replicated in $3 \mathrm{~B}, F<1$. The interaction between interference and phonological similarity was not significant in any of the three replications.

Planned comparisons for recall of the block-2 nonrhyming trials showed that there was no difference in recall between when the first block contained rhyming or non- rhyming words in $3 \mathrm{~A}$ and $3 \mathrm{~B}$, but there was a significant difference in $3 \mathrm{C}, F(1,19)=13.53, M S E=0.001, p=$ 0.002 . With rhyming items in the second block there was a significant decrement in the Rhyming words-Rhyming words condition in $3 \mathrm{~A}, F(1,19)=12.94, \eta^{2}=0.403$, $M S E=0.419, p=0.002$, and no effect in $3 \mathrm{~B}$ and $3 \mathrm{C}$.

\section{Block-1 intrusions}

Figure 4 and Table 1 indicate that in all three replications of the experiment there was a substantial increase in block-1 intrusions when the items in the two blocks all rhymed with each other. The difference was highly significant in all three versions of the experiment, 3A: $Z=3.62, p<0.001,3 \mathrm{~B}: Z=2.81, p=0.005$, and $3 \mathrm{C}$ : $Z=3.10, p=0.002$.

In the lower panel of Fig. 3 we plotted the within-list order errors for the Rhyming words-Rhyming words and Nonrhyming words-Rhyming words trials and add the block-1 intrusions for the Rhyming words-Rhyming words lists. Again, these intrusions do not fall on the projection of the within block order errors.

The three versions of the experiment were conducted to ensure that the central tenets of the Embedded Processes model were testable, that is, that items in the second block were well registered in the FOA and that items from the first block were no longer in the FOA. There are a number of indices that these manipulations were successful. No participant ever read the block-2 items aloud and in Experiment $3 \mathrm{~B}$ all participants complied with the instruction to say "beep". We take this as an indicator that people were following instructions and were attending to the items in the second block. The absolute levels of performance also suggest attention to those items. In looking at the non-rhyming conditions where recall is not influenced by phonological similarity, correct recall on the second block is very near ceiling if not on it $(3 \mathrm{~A}=0.92,3 \mathrm{~B}=0.92,3 \mathrm{C}=0.97)$. In addition, performance is better than that obtained in the four-item non-rhyming one-block trials where there is no interference possible $(3 \mathrm{~A}=0.86,3 \mathrm{~B}=0.86,3 \mathrm{C}=0.92)$. For the conditions containing rhyming items, the same pattern is true with better recall on the two-block trials $(3 \mathrm{~A}=0.89$, $3 \mathrm{~B}=0.89,3 \mathrm{C}=0.89)$ than the one-block trials $(3 \mathrm{~A}=$ $0.72,3 \mathrm{~B}=0.78,3 \mathrm{C}=0.84$ ) even though performance is off ceiling. In addition, if one looks at the very first serial position in the conditions where non-rhyming items are being recalled, performance is indistinguishable between the two-block trials (95\% correct) and the one-block trial $(93 \%$ correct). In other words, there does not seem to be a lot of evidence supporting the notion that people fail to switch attention or pay less attention on the second block in two-block trials. 
The results are again consistent in showing a phonological similarity effect when the items in the second block (and in one-block trials) all rhyme as reflected in both a decrement in correct-in-position scoring and an increase in transposition errors. Interference effects in the correct-inposition scores were more variable across the replications, a finding that is often observed in the cued recall experiments as well (Tehan \& Humphreys, 1998; Tolan \& Tehan, 2002). This is not surprising given that correct recall is influenced by other sources of error besides PI (e.g. omissions, momentary lapses of concentration, repetitions, phonological and other intrusions, etc.) and that even very rare sources of error are likely to have a larger impact on scoring when performance is near ceiling.

The strong test of PI is reflected in the number of block1 intrusions and here the data are very clear. When the items in block-1 and block-2 rhyme with each other there is an increased likelihood that the block-1 items will be incorrectly recalled. This was true of all three replications and was not limited to a small subset of participants making many errors. Thus, PI is readily evident in the rhymingrhyming condition.

\section{General discussion}

Our research dealt with the empirical issue of PI in shortterm memory tasks as a window for testing two current approaches to short-term recall. A key finding which the FOA approach emphasises is the change in PI effects that occur with changes in list length, with immunity to PI being observed at around four items and below and PI being observed at list lengths greater than four (Halford et al., 1988; Cowan et al., 2005). The alternative cue plus code approach argues that it is not list length per se that determines PI effects rather it is whether or not there is a source of information available that provides ease of discrimination among potential competitors. In the shortterm domain, that information is deemed to be phonological in nature.

Without rehashing the specifics of each approach, it is clear that our data are consistent with the cue plus code approach in that in all experiments both PI and immunity to PI are observed within the same list length, and the effects do not change with changes in list length. These results are predictable from a perspective that phonological codes can provide a source of discriminative information (Tehan \& Humphreys, 1995, 1998).

Our experiments represent a direct and straightforward test of the key assumptions of the embedded processes model that immunity to PI and the presence of PI should be observed as a function of list length. The data are not consistent with notions of direct access to a limited capacity store of four items. ${ }^{1}$ There have been a number of modifications made to the assumptions of the FOA approach that might still account for the data. First, the PI effects might reflect individual differences in the capacity of the FOA with three items being at or beyond capacity for a small number of participants. The data in Table 1 suggest that this explanation is not tenable. For example, in Experiment 3, very few participants make any intrusions in three of the conditions, yet $70 \%$ of participants make at least one intrusion error in the rhyming-rhyming condition. PI is not limited to a small minority of participants skewing the data.

Second, it has been suggested that participants can zoom attention in and out thereby changing the functional capacity of the FOA. We cannot rule this out as a posthoc explanation but would make the point that there does not appear to be a principled explanation for why the FOA should zoom out to a capacity of five items in those conditions showing immunity to PI in Experiment 2 and then zoom in to a capacity of less than three to explain the presence of PI in Experiment 3.

Third, it might be the case that items in the second block are not attended to sufficiently to ensure appropriate levels of encoding of the block-2 items either by a failure to shift attention from block-1 to block-2 or by not concentrating on the block-2 items. The three conditions in Experiment 3 addressed this issue and all aspects of the data converge on the idea that the items were attended to.

Differential encoding might still explain the additional decrement in correct recall on the rhyme-rhyme trials in Experiments 2 and 3A. Farrell and Lewandowksy (2002) propose a model of serial recall in which storage strength is determined by a similarity based attention mechanism. The basic assumption of this model is that if an item being presented for memory was similar to the items that preceded it, less attention would be paid to it. Thus in a list of similar items, less and less attention would be paid to

\footnotetext{
${ }^{1}$ Oberauer $(2002 ; 2005)$ has proposed an extension of the embedded processes model. In this model items in LTM are organised in terms of a network of associatively related nodes in which activation of one node can spread throughout the network and partially activate other related nodes. The equivalent of the FOA in this model is termed the direct-access region. This region is limited in capacity where capacity is represented as a limited number of temporary episodic bindings among nodes, rather than a limited number of items per se. Oberauer does have a FOA in the model but its capacity is seen as one item. In addition, in the differences in architecture, Oberauer does have a mechanism, at least in terms of recognition memory, where information from outside the direct-access region can still exert an influence upon performance. Our understanding of that model is that it has the potential to explain the serial recall data in Experiments 2 and 3 under certain circumstances, but would less readily account for the results in Experiment 1. We have not discussed this model here because the focus of our paper is on capacity estimates in terms of items not episodic bindings.
} 
the latter items in the list. In applying this mechanism to the rhyme-rhyme condition, the degree of attention paid to the second block items would be reduced due to prior study of similar items in the first block. Consequently, the theory predicts that the diminished encoding would lead to a decrement in recall of the block-2 items. Incorporating such a mechanism within the embedded processes model might conceivably lead to poor registration in the FOA and might lead to decreased levels of recall, but it does not explain the increase in block-1 intrusions from outside the FOA, nor does it account in any way for the cued recall data in Experiment 1.

Another possibility that has been suggested based upon both behavioural and neuro-imaging is that the capacity of the FOA is limited to a single item, not four as the embedded processes model assumes (Jonides et al., 2008; McElree, 2001; Oberauer, 2002; Verhaeghen \& Basak, 2005). If such is the case then the current experiments have little to say on the issue save that the absence of PI must be explained by other factors besides the FOA.

The embedded process model is appealing in that it is a general model of short-term cognition that is applicable across a range of short-term recognition, serial recall, cued recall and other working memory tasks. In addition, it makes principled predictions about memory performance that are independent of specific tasks. That is, factors involving modality of presentation or mode of responding are assumed to have only minimal impact upon memory performance. The similarity of results across tasks and across a range of input and output conditions indicates that these peripheral factors are not overly important in affecting PI as both the embedded processes and cue-plus-code models would argue.

However, one consequence of our results for the Embedded Processes account is that manipulating PI in short lists cannot provide a "pure" measure of the capacity of the FOA. Instead, our findings complement similar research showing that standard short-term retention and working memory tasks bear the hallmark of contributions from LTM (Nairne, 1990; Schweickert, 1993; Unsworth \& Engle, 2007). The PI effects reported here seem to be more amenable to explanations based upon more general principles of memory that apply over all retention intervals (e.g. cue based retrieval, trace discrimination, supporting codes, etc.) (Surprenant \& Neath, 2009).

\section{References}

Atkinson, R. C., \& Shiffrin, R. M. (1968). Human memory: A proposed system and its control processes. In K. W. Spence \& J. T. Spence (Eds.), The psychology of learning and motivation: Advanced in research and theory (Vol. 2). New York: Academic Press.
Baddeley, A. D. (1986). Working memory. Oxford: Oxford University Press.

Baddeley, A. D., \& Hitch, G. (1974). Working memory. In G. H. Bower (Ed.), The psychology of learning and motivation: Advances in research and theory (Vol. 8). New York: Academic Press.

Baddeley, A. D., Thomson, N., \& Buchanan, M. (1975). Word length and the structure of short-term memory. Journal of Verbal Learning and Verbal Behavior, 14, 575-589. doi:10.1016/ S0022-5371(75)80045-4

Brannelly, S., Tehan, G., \& Humphreys, M. S. (1989). Retrieval plus scanning: Does it occur? Memory \& Cognition, 17, 712-722.

Carroll, L. M., Jalbert, A., Penney, A. M., Neath, I., Surprenant, A. M., \& Tehan, G. (2010). Evidence for proactive interference in the focus of attention in working memory. Canadian Journal of Experimental Psychology, 64, 208-214. doi:10.1037/a0021011

Cowan, N. (1995). Attention and memory: An integrated framework. Oxford University Press.

Cowan, N. (1999). An embedded processes model of working memory. In A. Miyake \& P. Shah (Eds.), Models of working memory: Mechanisms of active maintenance and executive control. New York: Cambridge University Press.

Cowan, N. (2001). The magical number 4 in short-term memory: A reconsideration of mental storage capacity. The Behavioral and Brain Sciences, 24, 87-185. doi:10.1017/S0140525X01003922

Cowan, N., Johnson, T. D., \& Saults, S. (2005). Capacity limits in list item recognition: Evidence from proactive interference. Memory, 13(3), 293-299. doi:10.1080/09658210344000206

Dempster, F. N., \& Cooney, J. B. (1982). Individual differences in digit span, susceptibility to proactive interference, and aptitude/ achievement test scores. Intelligence, 6, 399-416. doi:10.1016/ 0160-2896(82)90026-

Estes, W. K. (1991). On types of item coding and source of recall in short-term memory. In W. E. Hockley \& S. Lewandowsky (Eds.), Relating theory and data: Essays on human memory in honor of Bennet B. Murdock (pp. 155-174). Hillsdale, NJ: Erlbaum.

Farrell, S., \& Lewandowsky, S. (2002). An endogenous model of ordering in serial recall. Psychonomic Bulletin \& Review, 9, 59-79.

Halford, G. S., Maybery, M. T., \& Bain, J. D. (1988). Set-size effects in primary memory: An age-related capacity limitation? Memory \& Cognition, 16, 480-487.

Hanley, M. J., \& Scheirer, C. J. (1975). Proactive inhibition in memory-scanning. Journal of Experimental Psychology, 104, 81-83. doi:10.1037/0278-7393.1.1.81

Henson, R. N. A. (1998). Short-term Memory for serial order: The start-end model. Cognitive Psychology, 36, 73-137. doi:10.1006/ cogp. 1998.0685

Humphreys, M. S., \& Tehan, G. (1992). A simultaneous examination of recency and cuing effects. In A. F. Healy, S. M. Kosslyn, \& R. M. Shiffrin (Eds.), From learning processes to cognitive processes: Essays in honor of William K. Estes (pp. 143-159). Hillsdale, NJ: Erlbaum.

Jonides, J., Lewis, R. L., Nee, D. E., Lustig, C. A., Berman, M. G., \& Moore, K. S. (2008). The mind and brain of short-term memory. Annual Review of Psychology, 59, 193-224. doi:10.1146/ annurev.psych.59.103006.093615

Jonides, J., Marshuetz, C., Smith, E. E., Reuter-Lorenz, P. A., Koeppe, R. A., \& Hartley, A. (2000). Age differences in behavior and PET activation reveal differences in interference resolution in verbal working memory. Journal of Cognitive Neuroscience, 12, 188-196. doi:10.1162/089892900561823

McElree, B. (2001). Working memory and focal attention. Journal of Experimental Psychology. Learning, Memory, and Cognition, 27, 817-835. doi:10.1037/0278-7393.27.3.817

Miller, G. A. (1956). The magical number seven, plus or minus two: Some limits on our capacity for processing information. Psychological Review, 63(2), 81-97. doi:10.1037/h0043158 
Monsell, S. (1978). Recency, immediate recognition memory, and reaction time. Cognitive Psychology, 10, 465-501. doi:10.1016/ 0010-0285(78)90008-7

Nairne, J. S. (1990). A feature model of immediate memory. Memory \& Cognition, 18(3), 251-269.

Oberauer, K. (2002). Access to information in working memory: Exploring the focus of attention. Journal of Experimental Psychology. Learning, Memory, and Cognition, 28, 411-421. doi:10.1037/0278-7393.28.3.411

Oberauer, K. (2005). Binding and inhibition in working memory: Individual and age differences in short-term recognition. Journal of Experimental Psychology: General, 134, 368-387. doi:10.1037/ 0096-3445.134.3.368

Sanders, A. F., \& Willemsen, E. M. (1978). The course of proactive interference in immediate probed recall. Acta Psychologica, 42, 133-144. doi:10.1016/0001-6918(78)90041-0

Schweickert, R. (1993). A multinomial processing tree model for degradation and redintegration in immediate recall. Memory \& Cognition, 21, 168-175.

Surprenant, A. M., \& Neath, I. (2009). Principles of memory. New York: Psychology Press.

Tehan, G., \& Humphreys, M. S. (1995). Transient phonological codes and immunity to proactive interference. Memory \& Cognition, 23 (2), 181-191.

Tehan, G., \& Humphreys, M. S. (1998). Creating proactive interference in immediate recall: Building a DOG from a DART, a MOP, and a FIG. Memory \& Cognition, 26(3), 477-489.

Tolan, G. A., \& Tehan, G. (1999). Determinants of short-term forgetting: Decay, retroactive interference, or proactive interference? International Journal of Psychology, 34(5/6), 285-292. doi:10.1080/002075999399585
Tolan, G. A., \& Tehan, G. (2002). Testing feature interaction: Between stream similarity effects in immediate recall. Journal of Memory and Language, 46, 562-585. doi:10.1006/ jmla.2001.2820

Tulving, E., \& Patterson, R. D. (1968). Functional units and retrieval processes in free recall. Journal of Experimental Psychology, 77, 239-248. doi:10.1037/h0025788

Unsworth, N., \& Engle, R. W. (2007). On the division of short-term and working memory: An examination of simple and complex span and their relation to higher order abilities. Psychological Bulletin, 133, 1038-1066. doi:10.1037/0033-2909.133.6.1038

Van Overschelde, J. P., Rawson, K. A., \& Dunlosky, J. (2004). Category norms: An updated and expanded version of the Battig and Montague (1969) norms. Journal of Memory and Language, 50, 289-335. doi:10.1016/j.jml.2003.10.003

Verhaeghen, P., \& Basak, C. (2005). Ageing and switching of the focus of attention in working memory: Results from a modified N-Back task. The Quarterly Journal of Experimental Psychology, 58A, 134-154. doi:10.1080/02724980443000241

Walling, J. R., McEvoy, C. L., Oth, J. E., \& Nelson, D. L. (1983). The University of South Florida rhyme category norms. Unpublished manuscript. University of South Florida.

Watkins, M. J. (1974). Concept and measurement of primary memory. Psychological Bulletin, 81, 695-711. doi:10.1037/ h0036952

Waugh, N. C., \& Norman, D. A. (1965). Primary memory. Psychological Review, 72, 89-104. doi:10.1037/h0021797

Wickens, D. D., Moody, M. J., \& Dow, R. (1981). The nature and timing of the retrieval process and of interference effects. Journal of Experimental Psychology: General, 110(1), 1-20. doi:10.1037/ 0096-3445.110.1.1 